\title{
CARCANGIU Bianca Maria. - Il territorio conteso. L'Ogaden etiopico e i Somali ogaadeen
}

\author{
Alain Gascon
}

\section{OpenEdition}

Journals

Édition électronique

URL : https://journals.openedition.org/etudesafricaines/30597

DOI : 10.4000/etudesafricaines.30597

ISSN : $1777-5353$

\section{Éditeur}

Éditions de l'EHESS

\section{Édition imprimée}

Date de publication : 15 juin 2020

Pagination : 445-447

ISBN : $978-2-7132-2828-5$

ISSN : 0008-0055

\section{Référence électronique}

Alain Gascon, " CARCANGIU Bianca Maria. - II territorio conteso. L'Ogaden etiopico e i Somali ogaadeen », Cahiers d'études africaines [En ligne], 238 | 2020, mis en ligne le 15 juin 2020, consulté le 04 janvier 2023. URL : http://journals.openedition.org/etudesafricaines/30597 ; DOl : https://doi.org/10.4000/ etudesafricaines.30597

Ce document a été généré automatiquement le 4 janvier 2023.

Tous droits réservés 


\title{
CARCANGIU Bianca Maria. - Il territorio conteso. L'Ogaden etiopico e $i$ Somali ogaadeen
}

\author{
Alain Gascon
}

\section{RÉFÉRENCE}

CARCANGIU Bianca Maria. - Il territorio conteso. L'Ogaden etiopico e i Somali ogaadeen.

Canterano, Aracne editrice, 2017, 206 p., bibl., index.

1 On aimerait lire plus souvent des livres qui, en si peu de pages, contribuent pleinement à la réflexion et à l'information. Il comporte une bibliographie classée (archives, articles, livres, etc.), en outre dans des langues autres que l'anglais, et un index complet. Ce court ouvrage comprend aussi un recueil de cartes qui aident à la compréhension, même si la première (p.7) est notoirement insuffisante. De plus, l'auteure reproduit des lettres en anglais de Sforza, ministre des Affaires étrangères d'Italie, qui réussit à obtenir, pour l'ex-puissance coloniale, le mandat des Nations Unies. Ainsi, l'Administration fiduciaire italienne de la Somalie (AFIS), installée en 1950, avait-elle la charge de mener l'ex-colonie à l'indépendance qui advint en 1960. Il manque, comme dans la plupart des publications sur les Somali ou la Somalie, la mention de l'orthographe officielle des toponymes et patronymes; mais il est vrai que l'étude se termine après la première guerre d'Ogadén (1963-1964) donc avant l'écriture officielle de la langue somali en caractères latins (1972).

2 La progression de la démarche de B. M. Carcangiu se décline en quatre chapitres d'inégale longueur qui examinent tour à tour le territoire contesté de l'Ogadén ${ }^{1}$ éthiopien et ses turbulents habitants. Le premier chapitre présente le territoire des Somali Ogaadeen, de la famille-clan Darood, des éleveurs musulmans qui nomadisent à la périphérie méridionale des hautes terres peuplées d'agriculteurs sédentaires chrétiens et musulmans d'Éthiopie. Le $2^{\mathrm{e}}$ chapitre traite de l'alternance des 
administrations en Ogadén depuis la conquête éthiopienne (fin XIX ${ }^{e}$ siècle), qui écarta les Européens de ce territoire, jusqu'à la guerre italo-éthiopienne (1935-1936) qui l'incorpora au bref Impèro. Après sa chute en 1941, l'Ogadén dépendit, jusqu'en 1954, de la British Military Administration. Le $3^{\mathrm{e}}$ chapitre, sans doute le plus ambitieux et le plus novateur, montre comment cette région enclavée et enfoncée en coin entre le Somaliland et l'ex-Somalia, disposant de maigres ressources et au peuplement clairsemé, devint une "monnaie d'échange» pour la diplomatie régionale puis internationale. Ces changements d'échelle nous font comprendre pourquoi le sort de bourgades, entourant des puits, tels Kelafo, Degah Bur ou Kebri Dehar, fut l'occasion d'échanger force notes entre les chancelleries, non seulement d'Éthiopie, de Somalie et des puissances coloniales ${ }^{2}$, mais également des deux Grands (URSS, États-Unis), d'Égypte, de la Ligue arabe, d'Inde, de l'ONU et de l'OUA fraîchement constituée. Le dernier chapitre, également de belle tenue, nous montre les réactions et les actions des Ogaadeen qui conduisirent à l'affrontement éthio-somalien de $1963-1964^{3}$ et à la naissance des fronts irrédentistes qui ont accepté, en août 2018, les offres de paix de l'Éthiopie d'Abiyy Ahmäd.

3 J'ai déploré 4 que, dans la Corne de l'Afrique, on pratique trop souvent les sciences sociales «zéro-sol », sans enracinement territorial, comme l'a regretté Luc Cambrézy. B. M. Carcangiu ne tombe pas dans ces errements, non seulement dans son premier chapitre de présentation, mais tout au long de son exposé. Elle associe à la notion, certes discutée, de "mode de production pastorale", le concept de "frontière pastorale " qui me paraît bien plus judicieux que la "pastoral democracy» de feu I. M. Lewis qu'on a, il est vrai, en quelque sorte dérobée à son auteur pour lui donner les acceptions les plus contradictoires. Ce concept nouveau aide ainsi à comprendre les difficultés rencontrées lors de la délimitation de frontières étatiques permanentes alors que chez les éleveurs prime la liberté de déplacement de leurs troupeaux et de leurs caravanes. B. M. Carcangiu insiste sur les deux visites officielles de Haylä Sellasé en 1935 et en 1954 afin de réaffirmer son pouvoir en Ogadén. Il réunit les chefs somali, les amadouant avec des prébendes et des titres tout en leur promettant de respecter leur identité, leurs coutumes, leurs parcours et leur commerce. Mais, une fois son pouvoir consolidé à l'issue de son deuxième voyage, l'administration impériale décida de percevoir un impôt sur les têtes de bétail. Un grand shir se réunit alors afin de résister, élargissant ainsi le champ d'application des shir, jusque-là des assemblées traditionnelles où se concluaient des pactes de voisinage (xeer) entre les segments de clans (reer) pour convenir de nouvelles frontières entre les terrains de parcours communs. On y fixait aussi le prix du sang (mag) afin de limiter les « retombées » des heurts inévitables qui surgissaient entre pasteurs.

4 Ce livre remet en question l'idée reçue, souvent ressassée, que les Somali - les Touaregs, les Toubous, les Peuls - étaient « par nature » rétifs à toute frontière et donc à tout État. J'avancerais plutôt, après bien d'autres, que les Somali, comme tous les pasteurs, voudraient fixer eux-mêmes, et pour eux-mêmes, les frontières d'autant qu'ils sont, eux et leur bétail, de plus en plus nombreux. L'auteure s'appuie sur un article pertinent de John Markakis ${ }^{5}$ qui traite de la réactivation des rivalités à propos du Hawd, un territoire traversé, au nord, par la frontière entre le Somaliland ex-britannique et l'Ogadén éthiopien. Pendant les pluies du gu (avril-juin), les troupeaux paissent dans les plateaux couverts de pâturages. Ils y demeurent grâce aux nombreuses mares qui subsistent pendant la courte saison sèche xagaa (juillet-septembre), jusqu'au retour des pluies de day (octobre-novembre). Jusqu'au retour du Hawd à l'Éthiopie (1955), les 
pâturages étaient fréquentés par les éleveurs du Somaliland des clans de la famille des Isxaaq, au nord, et, après 1955, par les Ogaadeen, au sud. Peut-être, l'auteure passe-telle un peu vite sur ces questions d'accès aux parcours pour le bétail, mais, près de 40 ans séparent les travaux de Markakis et la période étudiée par l'auteure. Dans la continuité de l'analyse des rivalités sur le territoire, elle nous montre comment les prospections pétrolières de la Sinclair Oil Co. ont été instrumentalisées par la GrandeBretagne, Haylä Sellasé, les chefs somali et l'AFIS et ont attiré l'attention des États-Unis.

Ce livre sur les Ogaadeen aide à mieux comprendre la fragilité de la Somalie, même réunifiée après 1960, ainsi que nous la présente Abdi Ismail Samatar ${ }^{6}$ dans sa biographie des deux hommes politiques qui lui ont assuré dix ans de démocratie dans un environnement chaotique. Si l'on veut comprendre, et apprécier, cette biographie double, parfois hagiographique, il faut absolument lire en contrepoint Il territorio conteso. L'Ogaden etiopico e i Somali ogaadeen. Cet ouvrage rappelle la profondeur historique des troubles qui affectent la péninsule somali et annonce également les fractures qui la traversent. Ainsi, le séparatisme somalilandais n'est pas uniquement le produit de la colonisation britannique, mais renvoie aux rivalités à propos des pâturages du Hawd encore exacerbées par l'utilisation du béton pour pérenniser les mares où s'abreuve le bétail, de plus exporté vif à la Mecque, pendant le hajj7 . La frontière dans le Hawd fait également vivre, outre les douaniers et les policiers, les boutiquiers et aussi... les contrebandiers ${ }^{8}$. Quand s'est ajoutée l'obligation d'utiliser, sous peine de sanctions sous la dictature de Siyaad Barre (1969-1991), le somali parlé au $\mathrm{Sud}^{9}$, on conçoit mieux combien est profond le morcellement de la péninsule somali et de sa population.

6 Espérons que ce livre, modeste par son format, mais ambitieux par son sujet, sera consulté non seulement par le happy few des somalisants, mais également par tous ceux qui travaillent sur les éleveurs somali et africains «en bout de piste » ainsi que le constatait, il y a 25 ans, Jean Gallais.

\section{NOTES}

1. L'Ogadén, territoire du clan des Ogaadeen, déborde les frontières éthiopiennes.

2. Wal-Wal, un puits précisément situé en Ogadén, fut le prétexte de l'agression italienne en 1935.

3. M. F ONTRIER, "La crise de 1963-1964 en Ogadén", Annales d'Éthiopie, 20, 2004, pp. 181-194.

4. A. GASCON, "L'Éthiopie et la Corne de l'Afrique, "hors-sol" ", Pount, 11, 2017, pp. 109-117.

5. J. MARKAKIS, " The Ishaq-Ogaden Dispute ", in A. H. AJ ORNÄS \& M. A. M. SAliH (dir.), Ecology and Politics: Environmental Stress and Security in Africa, Uppsala, Nordiskafrika Institutet, 1989, pp. 157-163. 
6. A. I. SAMATAR, Africa's First Democrats. Somalia's Aden A. Osman and Abdirazak H. Hussen, Bloomington-Indianapolis, Indiana University Press, 2016.

7. G. PINAULDT, L'or vivant des Somalis : des frontières, des troupeaux et des hommes face à la mondialisation des normes, Thèse de doctorat, Paris, Université Paris 8, 2014.

8. M. DJAMA, «Trajectoire du pouvoir en pays somali », in M. DJAMA \& A. GASCON (dir.), «La Corne dans tous ses États », Cahiers d'Études africaines, XXXVII (2), 146, 1997, pp. 403-428.

9. M. HAJI MUKHTAR, « Language Marginalisation, Ethnic Nationalism and Cultural Crisis in Somalia ", in M. V. HøHNE \& V. LuLING (eds.), Milk and Peace, Drought and War. Somali Culture, Society and Politics. Essays in Honour of I. M. Lewis, London, Hurst \& Co., 2010, pp. 279-300. Voir aussi mon compte rendu : A. GAscon, « Milk and Peace, Drought and War. Somali Culture, Society and Politics. Essays in Honour of I. M. Lewis ", Cahiers d'Études africaines, 217, 2015, <http://journals.openedition.org/etudesafricaines/18056>. 